\title{
Ability of Emergency Physicians to Detect Early Ischemic Changes of Acute Ischemic Stroke on Cranial Computed Tomography
}

\author{
Erden Erol Ünlüer ${ }^{a}$ Erdem Yaka ${ }^{c}$ Galip Akhan ${ }^{b}$ Önder Limon ${ }^{a}$ \\ Pinar Hanife Kara ${ }^{a}$ Özcan Yavaşi ${ }^{a} \quad$ Nergiz Vandenberk ${ }^{a}$ Yasemin Ece Nazli ${ }^{a}$ \\ Kürşad Kutlukc \\ Departments of a Emergency Medicine and ${ }^{b}$ Neurology, Izmir Ataturk Research and Training Hospital, and \\ 'Department of Neurology, Faculty of Medicine, Dokuz Eylul University, Izmir, Turkey
}

\section{Key Words}

Emergency physician · Stroke $\cdot$ Early ischemic changes ·

Computed tomography

\begin{abstract}
Objective: The objective of this study was to evaluate the ability of emergency physicians (EPs) to diagnose early ischemic changes due acute ischemic stroke on cranial computed tomography (CT). Subjects and Methods: Three EPs interpreted CT scans obtained within $3 \mathrm{~h}$ of symptom onset in 50 patients with acute stroke. The CT scans were interpreted by the EPs and compared to official neuroradiologist reports as a gold standard. $\kappa$ statistics were calculated to determine agreement among the three readers. Sensitivities and specificities were analyzed for each reader. Results: The EPs' sensitivities were 50,45.5, and 45.5\%, and specificities were 64.3, 82.1 , and $64.3 \%$, respectively. Focal parenchymal hypodensity was the criterion for which the EPs were the most sensitive $(77.3 \%)$. The ability of EPs to recognize early ischemic changes on CT scans in acute ischemic stroke was moderate based on sensitivities. Conclusion: Based on this study, EPs must be trained especially for recognizing early ischemic changes in acute ischemic stroke to improve their accuracy of interpretation.

Copyright $\odot 2012$ S. Karger AG, Basel
\end{abstract}

\section{Introduction}

Acute ischemic stroke (AIS) is one of the most common diseases among patients presenting to the Emergency Department with altered mental status and neurologic deficits. Intravenous tissue-plasminogen activator (i.v. rt-PA) has been the only approved treatment in AIS since 1996. Later that year, the National Institute of Neurologic Disorders and Stroke (NINDS, a branch of the National Institutes of Health) convened a consensus conference on rapid identification and treatment of AIS, setting goals for stroke care in the United States. Head computerized tomography (CT) is still an important diagnostic modality for patients with suspected AIS and is often used before starting i.v. rt-PA within $3 \mathrm{~h}$ of ischemia $[1,2]$. In the NINDS rt-PA Stroke Trial, the CT scan was used primarily to rule out intracranial hemorrhage (ICH) or other unexpected pathology that might masquerade as acute stroke. However, secondary analysis of the NINDS data and experience with the use of i.v. rt-PA $3 \mathrm{~h}$ after ischemic onset, most notably from the European Cooperative Acute Stroke Trial (ECASS), suggest that CT findings of advanced ischemia were associated with increased risk of ICH. Also, in ECASS, more subtle changes involving $>33 \%$ of the middle cerebral artery (MCA) territory have

\section{KARGER}

Fax +4161306 1234

E-Mail karger@karger.ch

www.karger.com
(C) 2012 S. Karger AG, Basel

1011-7571/12/0216-0534\$38.00/0

Accessible online at:

www.karger.com/mpp
Erden Erol Ünlüer

Department of Emergency Medicine

Izmir Ataturk Research and Training Hospital

TR-35360 Izmir (Turkey)

Tel. +90 232244 4444, E-Mail erolerdenun@yahoo.com 
caused increased numbers of ICH $[3,4]$. The ECASS investigators also suggest that subtle changes involving $<33 \%$ of the MCA territory might help identify the best candidates for thrombolysis [5]. Signs of stroke can appear on noncontrast head CT within $6 \mathrm{~h}$ of symptom onset, and retrospective analysis of the NINDS study showed that $30 \%$ of the initial head CTs showed ischemic changes $[6,7]$. Hyperdense MCA sign, loss of discrimination between gray and white matter in the basal ganglia, focal hypoattenuation, obscuration of the lentiform nucleus, cortical sulcal effacement, loss of the insular ribbon, and obscuration of the sylvian fissure compared to the contralateral hemisphere can be interpreted as early ischemic changes [8]. These CT findings were used to define and quantify early ischemic changes by the Alberta Stroke Program Early CT Score [9]. Although the presence of early ischemic changes on $\mathrm{CT}$, regardless of their extent, is not a contraindication to treatment, it has been shown that detecting early ischemic changes within the early period of AIS is important for determining a patient's prognosis and complications $[8,10]$. Because of the critical importance of excluding ICH and recognizing early ischemic changes on non-contrast $\mathrm{CT}$, there is apprehension regarding the ability of emergency physicians (EPs) to correctly interpret CT scans in hyperacute stroke patients. Recent analysis showed that ICH could be missed by treating physicians and the EPs' interpretations were determined to be poor to fair compared to neuroradiologists' interpretations [11, 12].

In this study, we aimed to determine the ability of EPs to interprete early ischemic changes on CT during AIS.

\section{Subjects and Methods}

The baseline noncontrast head CTs of 50 patients presenting with stroke from January 2007 to January 2008 were obtained from the medical records. All the baseline CTs were taken within $3 \mathrm{~h}$ of symptom onset, and the patients were then diagnosed with AIS. The CT scans were performed on a spiral CT with one channel (Siemens Somatom AR). Section thickness was $2.5-3.5 \mathrm{~mm}$ through the posterior fossa and $5-10 \mathrm{~mm}$ for the supratentorial regions of the cerebral hemispheres.

Three EPs working in our research and training government hospital with an average of 3 years of experience after completion of residency training for emergency medicine were unblinded to clinical information of the patients and asked to interpret the CT scans for intracranial pathology. This took $1 \mathrm{~h}$ overall. The EPs' interpretations were compared to official neuroradiologist reports (as gold standard). The Ethics Committee at our tertiary care hospital approved the study protocol.

Sensitivity and specificity were calculated for each EP compared to the gold standard using 95\% confidence intervals. Sensi-
Table 1. Sensitivity of early ischemic CT findings and 95\% confidence intervals (CI)

\begin{tabular}{|c|c|c|}
\hline Early ischemic changes & Sensitivity & $95 \% \mathrm{CI}$ \\
\hline Hyperdense MCA & 27.3 & $10.7-50.2$ \\
\hline $\begin{array}{l}\text { Shadowing or effacement of } \\
\text { lentiform nucleus }\end{array}$ & 22.7 & $7.8-45.4$ \\
\hline Loss of insular ribbon sign & 27.8 & $10.7-50.2$ \\
\hline Effacement of sylvian fissure & 36.4 & $17.2-59.3$ \\
\hline Effacement of cortical sulci & 63.6 & $40.7-82.8$ \\
\hline Focal parenchymal hypodensity & 77.3 & $54.6-92.2$ \\
\hline $\begin{array}{l}\text { Loss of gray-white differentiation } \\
\text { at basal ganglia }\end{array}$ & 36.4 & $17.2-59.3$ \\
\hline
\end{tabular}

tivities and specificities were calculated and the areas under receiver operating characteristic (ROC) curves were compared to each other for each early ischemic change. We analyzed all six early ischemic finding ROC curves in one graphic. Because the EPs' sensitivities in detecting hyperdense MCA, loss of insular ribbon, obscuration of the sylvian fissure, and loss of discrimination of gray and white matter in the basal ganglia findings on CT were equal, we showed both of them as a single line in figure 1.

The description of interobserver agreement included agreement rates and $\kappa$ of the EPs' interpretations of the CT scans compared to the gold standard. A multiple observer $\kappa$ coefficient with standard error was calculated for a dichotomous rating according to Fleiss [13] for all three observers combined. We defined $\kappa=$ $0.41-0.60$ as moderate agreement, $\kappa<0.40$ as poor agreement, and $\kappa>0.80$ as excellent agreement. Statistical analysis was performed using MedCalc ${ }^{\circledR}$ v11.2.1 and Matlab 7.1 pocket program at a $95 \%$ confidence level with expected $\mathrm{p}$ values lower than 0.05 .

\section{Results}

Of these 50 patients, $50 \%$ were female and their mean age was $72.86 \pm 12.36$ years (range of 39-90). The frequencies of the types of abnormalities found were as follows: effacement of the cortical sulci: $26 \%$; focal parenchymal hypodensity: $26 \%$; effacement of the sylvian fissure: $16 \%$; hyperdense MCA: $12 \%$; loss of insular ribbon sign: $12 \%$; loss of gray-white matter distinction in the basal ganglia: $12 \%$, and effacement or shadowing of the lentiform nucleus hypodensity: $8 \%$.

The sensitivities with confidence intervals for each of the possible early CT changes detected by EPs are listed in table 1 and ROC curves of each of the early ischemic changes are shown in figure 1 . The calculated sensitivities for interpreting early ischemic changes on noncontrast CT of each EP were 50, 45.5 and 45.5\% (28.2-71.8, 24.4$67.8,24.4-67.8$, respectively), while those of specificities 


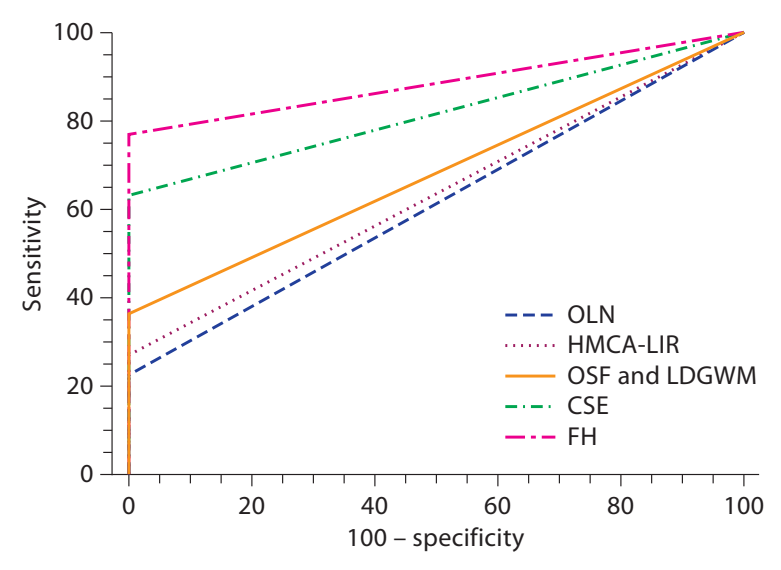

\begin{tabular}{|l|l|l|}
\hline & AUC & SE \\
\hline OLN & 0.614 & 0.0812 \\
\hline HMCA-LIR & 0.636 & 0.0801 \\
\hline OSF and LDGWM & 0.682 & 0.0774 \\
\hline CSE & 0.818 & 0.0629 \\
\hline FH & 0.886 & 0.0509 \\
\hline
\end{tabular}

Fig. 1. ROC curves of early ischemic changes. OLN = Obscuration of the lentiform nucleus; HMCA = hyperdense MCA sign; LIR = loss of the insular ribbon; OSF = obscuration of the sylvian fissure; LDGWM = loss of discrimination of gray and white matter in the basal ganglia; $\mathrm{CSE}=$ cortical sulcal effacement; $\mathrm{FH}=$ focal hypoattenuation; $\mathrm{AUC}=$ area under the curve; $\mathrm{SE}=$ standard error. Since the sensitivity values of HMCA and LIR are equal, they have the same ROC curve.

were $64.3,82.1$ and $64.3 \%(44.1-81.4,63.1-93.9,44.1-81.4$, respectively). There was good agreement beyond chance between the EPs based on the diagnostic accuracy for early ischemic changes ( $\mathrm{kappa}=0.37 ; 0.21-0.53$ ).

\section{Discussion}

The main finding of this study was that although early CT changes of ischemia may be present within $3 \mathrm{~h}$ of stroke symptom onset, agreement among EPs in recognizing them was moderate at best. These results are consistent with previous studies that have shown that nonradiologists and others without specific expertise in stroke, or even physicians experienced in stroke, may miss subtle changes or hemorrhage on CT [10-12]. Even among neuroradiologists with expertise in evaluating diagnostic studies of acute stroke patients, interobserver agreement that was based on a $78 \%$ prevalence of early CT changes was reported as moderate, with a $\kappa$ statistic of 0.53 [5].
Both the observed and reported moderate accuracy in identifying early ischemic changes could be due to the fact that CT does not detect blood degradation products such as hemosiderin and equally cannot identify patients with old brain hemorrhages [11].

Equally, calcification of the basal ganglia can occasionally be mistaken for deep intraparenchymal hemorrhage by EPs. Space-occupying lesions may also mimic ischemic stroke on CT.

Our finding that EPs could not identify early ischemic changes in CT scans either in the presence or absence of hemorrhage could indicate a lack of necessary skill for such interpretations. Hence these EPs will need specialized training to interpret CT scans with sufficient sensitivity to determine whether or not thrombolytics can be administered safely [11].

Interobserver agreement is not entirely explainable on the basis of experience and expertise because among the stroke specialists who were principal investigators in the NINDS rt-PA Stroke Trial and who each had several years' experience reading CT scans in hyperacute stroke patients, interobserver agreement was only fair [10]. Sensitivity for the identification of early signs of stroke is variable. The more apparent acute infarctions were identified by most physicians; however, even the best performers failed to identify a third of the more subtle acute infarctions.

Multiple studies following NINDS have shown an increased risk of $\mathrm{ICH}$, poor neurologic outcomes, and death in patients with early ischemic changes on head CT [14, 15]. Ischemic changes are relative contraindications to rtPA administration, and their presence may suggest that more than $3 \mathrm{~h}$ have elapsed from symptom onset, in which case systemic rt-PA may be contraindicated. In addition, the Food and Drug Administration, American Heart Association, and American Academy of Neurology specifically discourage administering i.v. rt-PA if early signs of major infarction are present, because of increased risk of ICH [16]. The greater the extent of ischemic changes on CT, the higher the risk of bleeding, as demonstrated in the ECASS-II [15]. In this setting, the most important job of the EPs in interpreting the CT is to rule out the presence of hemorrhage. Second, in the presence of significant unilateral neurologic abnormalities, hyperdense MCA sign should be sought. Third, early changes in CT should be identified, again using the patients' clinical symptoms to direct us to the likely abnormal side of the brain. These early changes may imply either an earlier time of onset than suggested by the history or a massive stroke in progress. 
The present study has several limitations. The optimal period for interpreting CT scans was not measured. However, our study was not designed to specifically address this issue. Other limitations of our study include the small number of interpreted CT scans, the relatively large confidence intervals and three EPs were not enough to clearly identify the performance in the Emergency Department. The performance of EPs was not compared with the control films based on the fact that EPs were blinded.

\section{Conclusion}

Our findings showed that EP performance in interpreting changes on CTs of patients with AIS was moderate and therefore recommend that they should be trained specifically on this topic after completing residency training for emergency medicine.

\section{References}

1 Culebras A, Kase CS, Masdeu JC, Masdeu CJ, Fox JA, Bryan NR, Grossman BC, Lee HD, Adams PH, Thies W: Practice guidelines for the use of imaging and transient ischemic attacks in acute stroke. A Report of the Stroke Council, American Heart Association. Stroke 1997;28:1480-1497.

-2 Keskin O, Kalemoğlu M, Ulusoy RE: A clinic investigation into prehospital and emergency department delays in acute stroke care. Med Princ Pract 2005;14:408-412.

- 3 Ingall TJ, O'Fallon MW, Asplund K, Goldfrank RL, Hertzberg SV, Louis AT, Christianson HJT: Findings from the reanalysis of the NINDS tissue plasminogen activator for acute ischemic stroke treatment trial. Stroke 2004;35:2418-2424.

4 Hacke W, Kaste M, Fieschi C, et al: Intravenous thrombolysis with recombinant tissue plasminogen activator for acute hemispheric stroke: The European Cooperative Acute Stroke Study (ECASS). JAMA 1995;274: 1017-1025.

5 Marks MP, Holmgren EB, Fox AJ, Patel S, Von Kummer R, Froehlich J: Evaluation of early computed tomographic findings in acute ischemic stroke. Stroke 1999;30:389392.
6 Scott PA, Barsan WG: Stroke, transient ischemic attack and other central focal conditions; in Tintinalli JE, Gelen GD, Stapczynski JS (eds): Emergency Medicine: A Comprehensive Study Guide, ed 5. McGraw-Hill, New York, 2000, pp 1430-1440.

7 Patel CS, Levine RS, Tilley CB, et al: Lack of clinical significance of early ischemic changes on computed tomography in acute stroke. JAMA 2001;286:2830-2838.

8 Kummer VR, Allen LK, Holle R, Bozzao L, Bastianello S, Manelfe C, Bluhmki E, Ringleb P, Meier DH, Hacke W: Acute stroke: usefulness of early CT findings before thrombolytic therapy. Radiology 1997;205:327-333.

-9 Barber PA, Demuchk AM, Zhang J, Buchan MA: Validity and reliability of a quantitative computed tomography score in predicting outcome of hyperacute stroke before thrombolytic therapy. ASPECTS Study Group. Alberta Stroke Programme Early CT Score. Lancet 2000;355:1670-1674.

10 Latchaw RE, Alberts MJ, Lev MH, et al: Recommendations for imaging of acute ischemic stroke: a scientific statement from the American Heart Association. Stroke 2009; 40:3646-3678.

11 Schriger DL, Kalafut M, Starkman S, Krueger M, Saver LJ: Cranial computed tomography interpretation in acute stroke: physician accuracy in determining eligibility for thrombolytic therapy. JAMA 1998;279:1293-1297.
12 Grotta CJ, Chiu D, Lu M, et al: Agreement and variability in the interpretation of early $\mathrm{CT}$ changes in stroke patients qualifying for intravenous rtPA therapy. Stroke 1999;30: 1528-1533.

13 Fleiss JL: Measuring nominal scale agreement among many raters. Psychol Bull 1971; 76:378-382.

14 Wardlaw JM, Mielke O: Early signs of brain infarction at CT: observer reliability and outcome after thrombolytic treatment: systematic review. Radiology 2005;235:444-453.

15 Dzialowski I, Hill MD, Coutts SB, Demchuk AM, Kent DM, Wunderlich O, von Kummer $\mathrm{R}$ : Extent of early ischemic changes on computed tomography (CT) before thrombolysis: prognostic value of the Alberta Stroke Program Early CT Score in ECASSII. Stroke 2006;37:973-978.

16 Adams HP Jr, Brott TG, Furlan AJ, et al: Guidelines for Thrombolytic Therapy for Acute Stroke: a Supplement to the Guidelines for the Management of Patients with Acute Ischemic Stroke. A statement for healthcare professionals from a Special Writing Group of the Stroke Council, American Heart Association. Stroke 1996;27:1711-1718. 